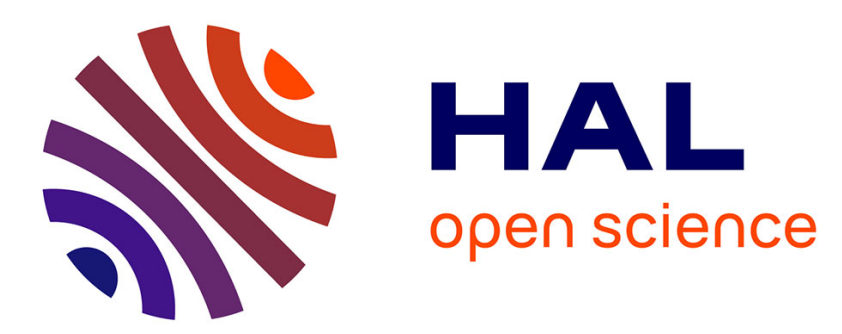

\title{
Finite Element Modelling Of Tensile Test For Micro-Alloyed Low Carbon Steel At High Temperature
}

Changli Zhang, Michel Bellet, Manuel Bobadilla, Houfa Shen, Baicheng Liu

\section{To cite this version:}

Changli Zhang, Michel Bellet, Manuel Bobadilla, Houfa Shen, Baicheng Liu. Finite Element Modelling Of Tensile Test For Micro-Alloyed Low Carbon Steel At High Temperature. Acta Metallurgica Sinica, 2010, 46 (10), pp.Pages 1206-1214. 10.3724/SP.J.1037.2010.01206 . hal-00570495

HAL Id: hal-00570495

https://hal-mines-paristech.archives-ouvertes.fr/hal-00570495

Submitted on 28 Feb 2011

HAL is a multi-disciplinary open access archive for the deposit and dissemination of scientific research documents, whether they are published or not. The documents may come from teaching and research institutions in France or abroad, or from public or private research centers.
L'archive ouverte pluridisciplinaire HAL, est destinée au dépôt et à la diffusion de documents scientifiques de niveau recherche, publiés ou non, émanant des établissements d'enseignement et de recherche français ou étrangers, des laboratoires publics ou privés. 


\title{
微合金低碳钢高温拉伸实验过程的有限元模拟 *
}

\author{
张长利 1) Michel Bellet ${ }^{2)} \quad$ Manuel Bobadilla ${ }^{3)}$ 沈厚发 1) 柳百成 ${ }^{1)}$ \\ 1) 清华大学机械工程系先进成形制造教育部重点实验室, 北京 100084 \\ 2) Mines-ParisTech, Centre de Mise en Forme des Matériaux (CEMEF), Sophia Antipolis, France 06904 \\ 3) ArcelorMittal, Research and Development, Maizières-lès-Metz, France 57283
}

\begin{abstract}
摘 要 开发了电势 - 热 - 力耦合有限元计算模型, 该模型考虑了微合金低碳钢高温条件下的奥氏体 $\gamma$ 和高温铁素体 $\delta$ 相变, 建立了多相混合力学模型, 描述了 $(\delta+\gamma)$ 两相混合区的力学行为. 应用多场耦合计算模型对拉伸实验过程进行了数值模拟. 实验 及数值模拟结果表明, 拉伸试样内存在较大径向及轴向温度梯度, 该温度梯度导致试样内产生相体积分数的梯度分布, 如高温铁素 体相分数和液相分数的梯度分布, 试样内产生非均匀变形, 并且应力分布极不均匀. 在进行实验钢高温力学本构方程的参数标定时, 采用名义应力 一 应变的方法会导致较大误差, 而基于数值模拟的方法则是十分有效及准确的, 如本文所建立的数值模型.
\end{abstract}

关键词 拉伸实验, 微合金低碳钢, 数值模拟

中图法分类号 TG142.12 文献标识码 A 文章编号 0412-1961(2010)10-1206-09

\section{FINITE ELEMENT MODELLING OF TENSILE TEST FOR MICRO-ALLOYED LOW CARBON STEEL AT HIGH TEMPERATURE}

\author{
ZHANG Changli ${ }^{1)}$, Michel Bellet ${ }^{2)}$, Manuel Bobadilla ${ }^{3)}$, SHEN Houfa ${ }^{1)}$, LIU Baicheng ${ }^{1)}$ \\ 1) Key Laboratory for Advanced Materials Processing Technology, Department of Mechanical Engineering, Tsinghua \\ University, Beijing 100084 \\ 2) Mines-ParisTech, Centre de Mise en Forme des Matériaux (CEMEF), Sophia Antipolis, France 06904 \\ 3) ArcelorMittal, Research and Development, Maizières-lès-Metz, France 57823 \\ Correspondent: SHEN Houfa, associate professor, Tel: (010)62789922, E-mail: shen@tsinghua.edu.cn \\ Supported by National Science \& Technology Major Project (No.2009ZX04014-082) \\ Manuscript received 2010-06-13, in revised form 2010-08-10
}

\begin{abstract}
In view of the numerical inverse identification of constitutive models, a forward numerical modelling of Gleeble tension tests is conducted. A coupled electrical-thermal-mechanical model is proposed for the resolution of electrical, energy and momentum conservation equations by means of finite element method. In momentum equation, the mixed rheological model in multi-phase region (e.g. $\delta$-ferrite and $\gamma$ austenite $(\delta+\gamma$ mixture) $)$ is developed to consider the $\delta / \gamma$ phase transformation phenomenon for micro-alloyed low carbon steel at high temperature. Experimental and numerical results reveal that significant thermal gradients exist in specimen along longitudinal and radial directions. Such thermal gradients will lead to phase fraction gradient in specimen at high temperature, such as $\delta$ fraction gradient or liquid fraction gradient. All these gradients will contribute to the heterogeneous deformation of specimen and severe stress non-uniform distribution, which is the major difficulty for the identification of constitutive models, especially for the simple identification method based on nominal stress-strain. The proposed model can be viewed as an important achievement for further inverse identification methods, which should be used to identify constitutive parameters for steel at high temperature in the presence of thermal gradients.
\end{abstract}

KEY WORDS Gleeble tension test, micro-alloyed low carbon steel, numerical modelling

\footnotetext{
* 国家科技重大专项资助项目 2009ZX04014-082

收到初稿日期：2010-06-13, 收到修改稿日期： 2010-08-10

作者简介：张长利, 男, 1976 年生, 博士生

DOI: $10.3724 /$ SP.J.1037.2010.00286
} 


\section{符号 说 明}

$A_{1}, A_{2}$
$C$
$E$
$F_{\text {cal }}$
$F_{\gamma, \text { cal }}$
$F_{\text {mush,cal }}$
$F_{\text {mix }, \text { cal }}$
$F_{\text {exp }}$
$H$
$\vec{I}$
$J_{\text {imp }}$
$\vec{J}$
$L$
$N_{\text {ph }}$
$P_{\text {int,elec }}$
$P_{\mathrm{v}, \text { elec }}$
$Q$
$R$
$S_{0}$
$S_{\gamma}$
$S_{\text {mush }}$
$S_{\text {mix }}$
$\vec{T}$
$\vec{V}$
$\vec{V}_{\mathrm{g}}$
$X_{\gamma}, X_{\text {mush }}, X_{\text {mix }}$
$a_{\varepsilon, \text { evp }}$
$a_{\dot{\varepsilon}, \text { evp }}, a_{\dot{\varepsilon}, \mathrm{vp}}$
$b$
$b_{\text {contact }}$

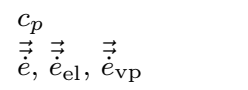

$f_{1}, f_{\mathrm{s}}$

$g_{i}$

$h_{\text {elec }}$

$h_{\text {th_eff }}$

$h_{\text {th_c }}$

$k$

$l_{0}$

m

$n$

$\vec{n}$

$p$

$r$

$r_{0}$ $\overrightarrow{\vec{s}}$
材料常数, $\mathrm{s}^{-1}$

$\mathrm{C}$ 的质量分数, \%

杨氏模量, MPa

计算的总拉伸载荷, $\mathrm{N}$

计算的奥氏体区域承受的拉伸载荷, $\mathrm{N}$

计算的糊状区承受的拉伸载荷, $\mathrm{N}$

计算的多相混合区承受的拉伸载荷, $\mathrm{N}$

实验测量的拉伸载荷, $\mathrm{N}$

焓, $\mathrm{J} / \mathrm{kg}$

单位张量

指定的电流密度, $\mathrm{A} / \mathrm{m}^{2}$

电流密度矢量, $\mathrm{A} / \mathrm{m}^{2}$

熔化潜热, $\mathrm{J} / \mathrm{kg}$

相的个数

界面接触电阻焦耳热功率, $\mathrm{W} / \mathrm{m}^{2}$

单位体积焦耳热功率, $\mathrm{W} / \mathrm{m}^{3}$

表观塑性变形激活能, $\mathrm{J} / \mathrm{mol}$

气体常数 $8.314 \mathrm{~J} /(\mathrm{mol} \cdot \mathrm{K})$

试样初始横截面积, $\mathrm{m}^{2}$

试样中心横截面上奥氏体区的面积, $\mathrm{m}^{2}$

试样中心横截面上糊状区的面积, $\mathrm{m}^{2}$

试样中心横截面上多相混合区的面积, $\mathrm{m}^{2}$

表面应力, $\mathrm{MPa}$

速度, $\mathrm{m} / \mathrm{s}$

夹具的移动速度, $\mathrm{m} / \mathrm{s}$

分别为奥氏体区、糊状区和多相混合区

所承受的载荷占总拉伸载荷的分数, $\%$

材料强化系数, MPa

材料常数, $\mathrm{MPa}$

材料的蓄热系数, $\mathrm{J} /\left(\mathrm{m}^{2} \cdot \mathrm{K} \cdot \mathrm{s}^{1 / 2}\right)$

相邻计算域接触面的材料蓄热系数,

$\mathrm{J} /\left(\mathrm{m}^{2} \cdot \mathrm{K} \cdot \mathrm{s}^{1 / 2}\right)$

比定压热容, $\mathrm{J} /(\mathrm{kg} \cdot \mathrm{K})$

分别为总应变速率、弹性应变速率及

黏塑性应变速率偏张量

液相和固相质量分数

第 $i$ 相的体积分数

等效界面导电系数, $\mathrm{A} /\left(\mathrm{m}^{2} \cdot \mathrm{V}\right)$

等效对流换热系数, $\mathrm{W} /\left(\mathrm{m}^{2} \cdot \mathrm{K}\right)$

接触界面等效换热系数, $\mathrm{W} /\left(\mathrm{m}^{2} \cdot \mathrm{K}\right)$

导热系数, $\mathrm{W} /(\mathrm{m} \cdot \mathrm{K})$

假设的试样均匀变形区长度, $\mathrm{m}$

应变速率敏感性指数

应变硬化指数

界面外法向矢量

压力, $\mathrm{MPa}$

试样半径, $m$

试样原始半径, $\mathrm{m}$

应力偏张量, $\mathrm{MPa}$
时间, $\mathrm{s}$

施加载荷时刻, $s$

第 $i$ 相的应力混合权函数

轴向单位矢量

材料常数, $\mathrm{MPa}^{-1}$

罚常数

总应变速率张量

弹性应变速率张量

热应变速率张量

黏塑性应变速率张量

相变体积应变速率张量

相变应变速率张量

等效塑性应变速率

电势, $\mathrm{V}$

相邻计算域的接触表面电势, $\mathrm{V}$

电导率, $\mathrm{S} / \mathrm{m}$

塑性乘子, $(\mathrm{MPa} \cdot \mathrm{s})^{-1}$

Lame 常数, MPa

Poisson 系数

温度, ${ }^{\circ} \mathrm{C}$

相邻计算域的接触表面温度, ${ }^{\circ} \mathrm{C}$

参考温度, ${ }^{\circ} \mathrm{C}$

环境温度, ${ }^{\circ} \mathrm{C}$

试样表面温度, ${ }^{\circ} \mathrm{C}$

密度, $\mathrm{kg} / \mathrm{m}^{3}$

轴向应力, $\mathrm{MPa}$

试样中心横截面上的

平均轴向应力, $\mathrm{MPa}$

根据实验拉伸载荷计算得到的

名义轴向应力, $\mathrm{MPa}$

计算的固相和糊状区

轴向应力, $\mathrm{MPa}$

等效应力, $\mathrm{MPa}$

应力张量, $\mathrm{MPa}$

在铸造过程中, 金属铸件内经常会形成各种铸造缺 陷, 其中包括热裂纹, 也称为凝固裂纹, 容易在承受拉应 力的凝固末期糊状区内形成. 热裂纹缺陷会恶化铸件力学 性能或加工性能, 且很难通过后续工艺消除. 为预测热裂 纹的形成, 文献 ${ }^{[1-4]}$ 报导了很多宏观热裂纹形成判据. 这 些判据表明, 热裂纹的形成与金属凝固过程中铸件内的热 - 力学状态有关, 如温度、应力、应变和应变速率等. 钢 在凝固及随后冷却过程中发生复杂的组织形貌及相转变, 因此, 预测钢在糊状区及临近熔点的固相区域内的应力应 变状态是十分复杂和困难的. 由于钢的高熔点, 因此研究 钢在高温（糊状区及近熔点）条件下的力学性能对实验装 置及方法提出了苛刻的要求. 浸入式分离凝固拉伸实验 (submerged split-chill tensile test) ${ }^{[5,6]}$ 能很好地模拟 凝固时合金的力学性能, 但其实验装置复杂, 且坯壳的凝 固生长及温度梯度使实验数据处理变得较为复杂和困难. 
在 Gleeble 热模拟试验机上对钢的高温力学性能进行实 验研究 ${ }^{[7-9]}$ 的方法简单易行, 因而得到了广泛应用. 然而 由于热传导及表面热损失, 拉伸试样内部存在一定的温度 梯度. 目前对试样内不均匀温度分布的处理方法, 只是简 单采用试样内和表面的平均温度来定义名义测试温度 ${ }^{[8]}$. 进行 Gleeble 实验时, 了解温度梯度对试样内应力一应 变分布特征的影响, 对研究钢在高温条件下的力学行为十 分必要.

针对金属铸造过程中铸件内通常产生小应变速率及 小应变, 本文采用了拉伸实验及数值模拟方法进行研究微 合金低碳钢在高温 $\left(1200{ }^{\circ} \mathrm{C}\right.$ 至糊状区)、小应变 $(<5 \%)$ 和 小应变速率 $\left(<10^{-2} \mathrm{~s}^{-1}\right)$ 条件下的力学行为. 以往文献 报道的热模拟实验的温度场数值模拟结果已经很好地指 导了各种试样的设计 ${ }^{[10-12]}$. 然而在数值模拟时很少同 时考虑热模拟实验中涉及到的电场、温度场及应力场，因 此, 本文的工作在于建立电势 - 热 - 力三场耦合计算模 型, 并考虑钢高温相变现象. 将该数值模型应用于微合金 低碳钢的拉伸实验过程的数值模拟, 为进一步确定本构方 程中材料参数的标定奠定基础

\section{1 实验方法}

实验所用微合金低碳钢的主要成分 (质量分数, \%) 为: $\mathrm{C} 0.065, \mathrm{Si} 0.2, \mathrm{Mn} 1.64, \mathrm{Al} 0.036, \mathrm{Nb} 0.064, \mathrm{Ti}$ $0.016, \mathrm{~S} 0.003, \mathrm{P} 0.012, \mathrm{Fe}$ 余量. 在连铸板坏上截取 并制备成直径 $10 \mathrm{~mm}$, 长 $120 \mathrm{~mm}$ 的棒状拉伸试样, 在 Gleeble1500D 试验机上进行高温拉伸实验. 用焊接在试 样表面中心的热电偶 (标记 thermocouple-0, 缩写 TC0) 来控制试样按预设的温度曲线加热. 在试样中心钻孔并放 置一付热电偶（标记 thermocouple-1, 缩写 TC1), 如图 1 所示. 此外, 为了解试样轴向温度的分布, 也测量了距 离试样表面中心 ( $\mathrm{TC} 0$ ) 处的温度值. 在下文中如未明确 提及, 所述温度均为 $\mathrm{TC} 0$ 处的温度值. 为表述方便, 本文 提及的 “轴线中心” 为 TC1 位置, “表面中心” 为 TC0 位置.

将试样按照图 2 所示的加热曲线加热至测试温度, 保 温 $1 \mathrm{~min}$ 后, 在 $t_{\mathrm{ms}}$ 时刻开始施加拉伸载荷. 为防止氧 化, 试样及夹具等放置在真空度约 $0.067 \mathrm{~Pa}$ 的真空箱内. 将试样用透明石英管包套, 以应对试样工作区可能发生的 熔化.

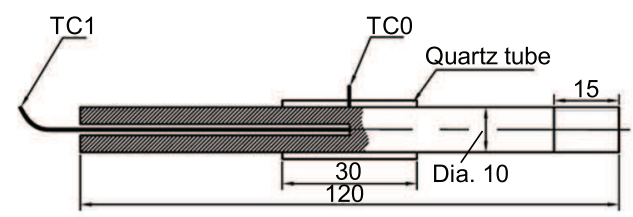

图 1 拉伸试样轴线中心温度测量示意图

Fig.1 Schematic of temperature measurements at axial center of tensile specimen (TC0 and TC1 are thermocouples)(unit: $\mathrm{mm}$ )

\section{2 有限元模型}

\section{1 几何模型}

根据拉伸试样及夹具的几何特点, 在有限元分析计算 时采用轴对称几何模型, 如图 3 所示. 夹具及试样的边界 也在图中标出.

\section{2 数学模型}

试样采用电阻加热, 电流密度可表示为 ${ }^{[13]}$

$$
\vec{J}=-\kappa \nabla \phi
$$

根据电流守恒, 可得到求解电势的 Poisson 方程

$$
\nabla \cdot(\kappa \nabla \phi)=0
$$

小变形速率时形变耗散能忽略不计. 考虑电阻热 $P_{\mathrm{v}, \text { elec }}$ 后, 能量方程为 $[13]$

$$
\rho \frac{\mathrm{d} H}{\mathrm{~d} t}=\nabla \cdot(\kappa \nabla \theta)=P_{\mathrm{v}, \text { elec }}
$$

$H$ 定义为

$$
H=\int_{\theta_{\mathrm{ref}}}^{\theta} c_{p}(t) \mathrm{d} t+f_{1} L
$$

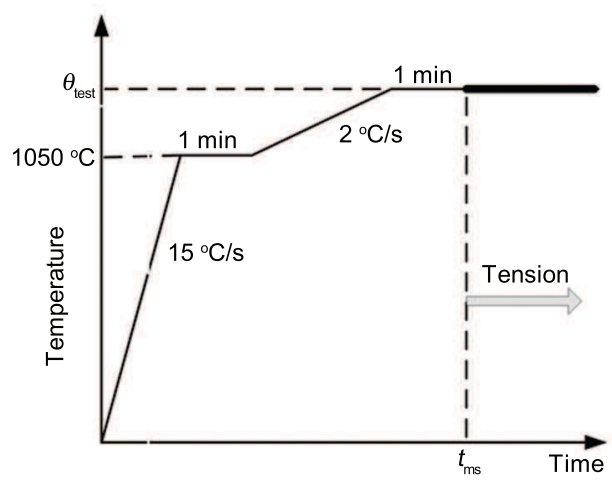

图 2 Gleeble 拉伸实验的加热曲线及载荷示意图

Fig.2 Schematic diagram of thermal-mechanical history of Gleeble tension test $\left(t_{\mathrm{ms}}\right.$ : the instant of starting mechanical loading; $\theta_{\text {test }}$ : the temperature at which tension test is conducted)

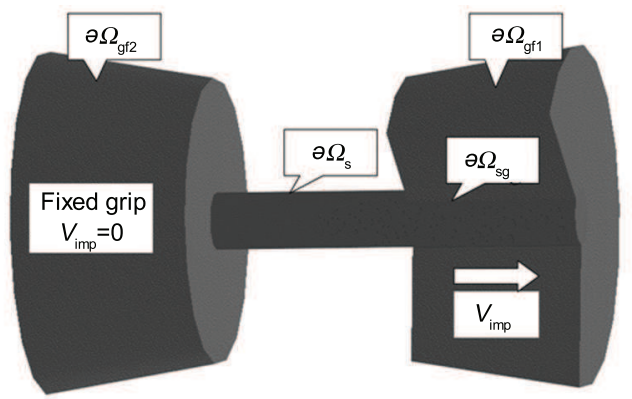

图 3 拉伸试样及夹具的轴对称几何模型

Fig.3 Schematic diagram of axi-symmetric geometrical model of tensile set-ups 
假定固、液相具有相同密度, 则质量分数和体积分数 相等, 因此各温度下的焓值均可以计算得到. 式 (3) 中的 电阻热由 Joule 定律得到

$$
P_{\mathrm{v}, \text { elec }}=\kappa \nabla \phi \cdot \nabla \phi
$$

为描述固相和糊状区力学行为, 本文采用文献 [14] 和 [15] 提出的固相和糊状区混合力学模型. 在温度高 于固相点的糊状区内, 采用热 - 黍塑性模型 (thermoviscoplastic, THVP) 来描述糊状区力学行为. 总应变 速率分解为热应变速率和黏塑性应变速率之和

$$
\overrightarrow{\dot{\varepsilon}}=\overrightarrow{\dot{\varepsilon}}_{\mathrm{vp}}+\overrightarrow{\dot{\varepsilon}}_{\mathrm{th}}
$$

式中, 黏塑性应变速率和热应变速率分别为

$$
\begin{gathered}
\overrightarrow{\vec{\varepsilon}}_{\mathrm{vp}}=\frac{3}{2 a_{\dot{\varepsilon}, \mathrm{vp}}} \dot{\bar{\varepsilon}}^{1-m} \overrightarrow{\vec{s}} \\
\overrightarrow{\dot{\varepsilon}}_{\mathrm{th}}=-\frac{1}{3 \rho} \frac{\mathrm{d} \rho}{\mathrm{d} t} \overrightarrow{\vec{I}}
\end{gathered}
$$

式 (8) 表示钢在液/固转变时的凝固收缩. 定义 Von Mises 等效应力及等效应变速率为

$$
\bar{\sigma}=\sqrt{\frac{3 \overrightarrow{\vec{s}}: \overrightarrow{\vec{s}}}{2}} \quad \dot{\bar{\varepsilon}}=\sqrt{\frac{2 \overrightarrow{\dot{\varepsilon}_{\mathrm{vp}}}: \overrightarrow{\dot{\varepsilon}}_{\mathrm{vp}}}{3}}
$$

由式 (7) 和 (9) 可以得到标量形式的黏塑性本构 方程

$$
\bar{\sigma}=a_{\dot{\varepsilon}, \mathrm{vp}} \dot{\bar{\varepsilon}}^{m}
$$

当温度低于固相点时, 采用热 - 弹 - 黏塑性模型 (thermo-elastic-viscoplastic, THEVP) 来描述钢在高 温下的力学行为. 总应变速率分解为

$$
\overrightarrow{\dot{\varepsilon}}=\overrightarrow{\dot{\varepsilon}}_{\mathrm{el}}+\overrightarrow{\overrightarrow{\dot{\varepsilon}}}_{\mathrm{vp}}+\overrightarrow{\dot{\vec{\varepsilon}}}_{\mathrm{th}}
$$

式中, 弹性应变速率和黏塑性应变速率分别为

$$
\begin{gathered}
\overrightarrow{\dot{\varepsilon}}_{\mathrm{el}}=\frac{1+v}{E} \overrightarrow{\overrightarrow{\dot{\sigma}}}-\frac{v}{E} \operatorname{tr}(\overrightarrow{\vec{\sigma}}) \overrightarrow{\vec{I}} \\
\overrightarrow{\vec{\varepsilon}}_{\mathrm{vp}}=\frac{3 \dot{\bar{\varepsilon}} \overrightarrow{\vec{s}}}{2 \bar{\sigma}}
\end{gathered}
$$

式 (13) 中等效应变速率和等效应力的关系由采用的 本构关系来确定. 忽略重力和惯性效应, 动量守恒方程为

$$
\nabla \cdot \vec{\sigma}=0
$$

\section{3 边界条件}

夹具的边界 $\partial \Omega_{\mathrm{gf} 1}$ 和 $\partial \Omega_{\mathrm{gf} 2}$ 分别指定零电势和电流 密度 $J_{\mathrm{imp}}$, 夹具和试样的接触界面 $\partial \Omega_{\mathrm{sg}}$ 的电流密度为

$$
\vec{J} \cdot \vec{n}=-J_{\mathrm{imp}}
$$

$$
\vec{J} \cdot \vec{n}=h_{\text {elec }}\left(\phi-\phi_{\text {contact }}\right)
$$

需要说明的是, 为模拟复杂的升温历史曲线, 采用 PID(proportional-integral-derivative) 算法在每个时 间步更新 $J_{\mathrm{imp}}$, 其基本原理是根据温度计算值 ( $\mathrm{TC} 0$ 位 置) 与预设值间偏差大小动态调节 $J_{\mathrm{imp}}$.

在拉伸试样表面 $\partial \Omega_{\mathrm{s}}$ 和夹具的表面 $\partial \Omega_{\mathrm{gf1} 1}$ 和 $\partial \Omega_{\mathrm{gf} 2}$, 采用等效对流散热条件

$$
-k \nabla \theta \cdot \vec{n}=h_{\mathrm{th} \_ \text {eff }}\left(\theta-\theta_{\mathrm{env}}\right)
$$

在接触界面 $\partial \Omega_{\mathrm{sg}}$ 上, 由于存在界面电阻热效应, 界 面热流可写为

$$
-k \nabla \theta \cdot \vec{n}=h_{\text {th_c }}\left(\theta-\theta_{\text {contact }}\right)+\frac{b}{b+b_{\text {contact }}} P_{\text {int }, \text { elec }}
$$

式中 $b=\sqrt{k \rho c_{\mathrm{p}}}, P_{\text {int }, \text { elec }}=h_{\text {elec }}\left(\phi-\phi_{\text {contact }}\right)^{2}$, 式 $(18)$ 表示界面电阻热在两接触部件的分配方式. 参数 $h_{\mathrm{th} \_ \text {eff }}$ 和 $h_{\mathrm{th} \_\mathrm{c}}$ 根据实际温度测量值由反算方法确定.

夹具假定为刚性零件. 在试样和夹具的接触界面, 设定双边黏结接触条件 (式 (19)) 或双边光滑接触条件 (式 $(20)$ )

$$
\begin{gathered}
\vec{V}-\vec{V}_{\mathrm{g}}=0 \\
\vec{T}=\overrightarrow{\vec{\sigma}} \vec{n}=-\chi_{\mathrm{p}}\left(\vec{V}-\vec{V}_{\mathrm{g}}\right) \\
\left(\vec{V}-\vec{V}_{\mathrm{g}}\right) \cdot \vec{n}=0 \\
\vec{T}_{n}=\overrightarrow{\vec{\sigma}} \vec{n}=-\chi_{\mathrm{p}}\left(\left(\vec{V}-\vec{V}_{\mathrm{g}}\right) \cdot \vec{n}\right) \vec{n}
\end{gathered}
$$

在实际实验中, 通常采用零载荷控制方法来降低试样内的 热应力, 消除对试样可能造成的破坏. 在载荷施加时刻 $t_{\mathrm{ms}}$ 之前, 应用双边光滑接触条件; 在 $t_{\mathrm{ms}}$ 时刻之后, 应用双边 黏结接触条件. 在数值模拟中, 采用这种边界条件的切换 来实现试样轴向的零载荷控制.

\section{4 有限元求解模型}

式 (2) 和 (3) 的弱解形式的方程为

$$
\begin{gathered}
\forall \phi^{*}: \quad \int_{\Omega} \kappa \nabla \phi \cdot \nabla \phi^{*} \mathrm{~d} \Omega+\int_{\partial \Omega} \phi^{*} \vec{J} \cdot \vec{n} \mathrm{~d} \Gamma=0 \\
\forall \varphi^{*}: \quad \int_{\Omega} \rho \frac{\mathrm{d} H}{\mathrm{~d} t} \varphi^{*} \mathrm{~d} \Omega+\int_{\Omega} k \nabla \theta \cdot \nabla \varphi^{*} \mathrm{~d} \Omega+ \\
\int_{\partial \Omega}-k \nabla T \cdot \vec{n} \varphi^{*} \mathrm{~d} \Gamma-\int_{\Omega} P_{\mathrm{v}, \operatorname{elec} \varphi^{*} \mathrm{~d} \Omega=0}
\end{gathered}
$$

式中, $\phi^{*}$ 和 $\varphi^{*}$ 为测试函数, 采用标准的 Galerkin 方法 在线性三角形离散单元上离散上述方程. 采用 NewtonRaphson 迭代方法求解式 (22) 离散后的方程, 刚度矩阵 包含了节点温度对焓的导数 $\partial \theta / \partial H$. 
采用速度/压力混合格式，式 (14) 的弱解形式方 程为

$$
\left\{\begin{array}{l}
\forall \vec{V}^{*}: \int_{\Omega} \overrightarrow{\vec{s}}: \overrightarrow{\vec{\varepsilon}}^{*} \mathrm{~d} \Omega-\int_{\Omega} p \nabla \cdot \overrightarrow{\vec{V}}^{*} \mathrm{~d} \Omega-\int_{\partial \Omega} \vec{T} \cdot \vec{V}^{*} \mathrm{~d} \Gamma=0 \\
\forall p^{*}: \int_{\Omega} p^{*} \operatorname{tr}\left(\overrightarrow{\dot{\varepsilon}}_{\mathrm{vp}}\right) \mathrm{d} \Omega=0
\end{array}\right.
$$

方程（23）中的第一个方程可由虚功原理得到，第 二个方程代表材料的不可压缩性. 将方程 (23) 采用 $\mathrm{P} 1+/ \mathrm{P} 1$ 格式在线性三角形单元上离散 ${ }^{[16]}$, 离散方程采 用 Newton-Raphson 方法求解 ${ }^{[17]}$.

\section{3 高温多相混合力学模型}

材料内发生相变时, 应变速率可分解为 ${ }^{[18]}$

$$
\overrightarrow{\vec{\varepsilon}}=\overrightarrow{\vec{\varepsilon}}_{\mathrm{el}}+\overrightarrow{\dot{\varepsilon}}_{\mathrm{vp}}+\overrightarrow{\dot{\varepsilon}}_{\mathrm{th}}+\overrightarrow{\dot{\varepsilon}}_{\mathrm{tr}}+\overrightarrow{\dot{\varepsilon}}_{\mathrm{pt}}
$$

为简化起见, 忽略相变应力引起的应变 $\overrightarrow{\dot{\varepsilon}}_{\mathrm{pt}}$. 当假设 各相密度相同时, 相变体积应变 $\overrightarrow{\vec{\varepsilon}_{\mathrm{tr}}}=0$, 从而式 $(24)$ 变为 式 (11)。

式 (23) 第二个方程中体积应变率在 THEVP 模型 时可写为:

$$
\begin{aligned}
& \operatorname{tr} \overrightarrow{\dot{\varepsilon}}_{\mathrm{vp}}=\operatorname{tr} \overrightarrow{\overrightarrow{\dot{\varepsilon}}}-\operatorname{tr} \overrightarrow{\dot{\varepsilon}}_{\mathrm{el}}-\operatorname{tr} \overrightarrow{\dot{\varepsilon}}_{\mathrm{th}}-\operatorname{tr} \overrightarrow{\dot{\varepsilon}}_{\mathrm{tr}}=\nabla \cdot \vec{V}+\frac{3(1-2\langle\nu\rangle)}{\langle E\rangle} \dot{p}+ \\
& \frac{1}{\langle\rho\rangle} \frac{\mathrm{d}\langle\rho\rangle}{\mathrm{d} t}-\sum_{i \rightarrow j} \frac{1}{3} \frac{\rho_{i}-\rho_{j}}{\rho_{j}} \dot{g}_{i \rightarrow j} \overrightarrow{\vec{I}}=0
\end{aligned}
$$

式中, $\langle E\rangle=\sum_{i=1}^{N_{\mathrm{ph}}} g_{i} E_{i},\langle\nu\rangle=\sum_{i=1}^{N_{\mathrm{ph}}} g_{i} \nu_{i},\langle\rho\rangle=\sum_{i=1}^{N_{\mathrm{ph}}} g_{i} \rho_{i}$, 为简化起见, 权函数均取为各相的体积分数 $g_{i}$, 因此算符 $\langle\cdot\rangle$ 代表体积平均值.

对应变偏量

$$
\overrightarrow{\dot{\vec{e}}}=\overrightarrow{\dot{\vec{e}}}_{\mathrm{el}}+\overrightarrow{\dot{\vec{e}}}_{\mathrm{vp}}
$$

式中

$$
\begin{array}{r}
\overrightarrow{\dot{\vec{e}}}_{\mathrm{el}}=\frac{1}{2\langle\mu\rangle} \overrightarrow{\overrightarrow{\dot{s}}}-\frac{1}{2\langle\mu\rangle^{2}} \frac{\mathrm{d}\langle\mu\rangle_{\overrightarrow{\vec{s}}}}{\mathrm{~d} t} \\
\left\{\begin{array}{c}
\langle\mu\rangle=\frac{\langle E\rangle}{2(1+\langle\nu\rangle)} \\
\overrightarrow{\dot{\vec{e}}}=\langle\dot{\lambda}\rangle \overrightarrow{\vec{s}}
\end{array}\right.
\end{array}
$$

式中塑性乘子为

$$
\langle\dot{\lambda}\rangle=\frac{3}{2} \frac{\dot{\bar{\varepsilon}}}{\bar{\sigma}}
$$

式 (29) 中 $\bar{\sigma}$ 为多相混合材料的等效应力. 由于各相 力学性质相异, 等效应力 $\bar{\sigma}$ 取决于各组成相的力学性质, 相分数及相分布形态等 ${ }^{[19]}$. 本文引入组成相的等效应力 加权函数 $w_{i}\left(g_{i}\right)$, 多相混合应力可写为

$$
\bar{\sigma}=\sum_{i}^{N_{\mathrm{ph}}} w_{i} \bar{\sigma}_{i}, \quad \text { 且 } \quad \sum_{i}^{N_{\mathrm{ph}}} w_{i}=1
$$

在实现多相混合力学模型时, 存在 2 个假设 ${ }^{[19]}$ : 等 应变假设和等应力假设. 等应变假设是指各相内应变相 等, 而等应力假设是指各相内应力相同. 对奥氏体相和 铁素体相混合力学模型, 等应变假设更符合实际材料性 能 ${ }^{[20,21]}$, 即: $\dot{\bar{\varepsilon}}_{i} \equiv \dot{\bar{\varepsilon}}$. 根据该假设, 奥氏体组成相的力 学行为可采用如下本构方程:

$$
\bar{\sigma}_{\gamma}=a_{\varepsilon, \mathrm{evp}} \bar{\varepsilon}^{n}+a_{\dot{\varepsilon}, \mathrm{evp}} \dot{\bar{\varepsilon}}^{m}
$$

参数的取值为 $[22]$

$$
\begin{gathered}
m=1 /\left(8.132-1.540 \times 10^{-3}(\theta+273)\right) \\
n=-0.6289+1.114 \times 10^{-3}(\theta+273) \\
a_{\varepsilon, \text { evp }}=130.5-5.128 \times 10^{-3}(\theta+273) \\
a_{\dot{\varepsilon}, \text { evp }}=\left(\frac{1}{A_{1}} \exp \left(\frac{Q}{R(\theta+273)}\right)\right)^{m}
\end{gathered}
$$

其中, $A_{1}=46550+71400 C+12000 C^{2}, \quad Q=$ $371.2 \mathrm{~kJ} / \mathrm{mol}$.

高温 $\delta$ - 铁素体组成相的力学行为采用如下本构 方程:

$$
\bar{\sigma}=\frac{1}{\alpha} \sinh ^{-1}\left[\frac{1}{A_{2}} \exp \left(\frac{m Q}{R(\theta+273)}\right) \dot{\bar{\varepsilon}}^{m}\right]
$$

参数取值为 ${ }^{[7]}: \alpha=0.0522 \mathrm{MPa}^{-1}, A_{2}=9.997 \times$ $10^{7} \mathrm{~s}^{-1}, Q=202.1 \mathrm{~kJ} / \mathrm{mol}, m=0.2657$.

根据应力在熔点的连续性, 由式 (32) 确定式 (10) 的参数 $a_{\dot{\varepsilon}, \mathrm{vp}}$ 和 $m$ 在固相点的数值为 $5.65 \mathrm{MPa}$ 和 $0.266 ;$ 在 $1510{ }^{\circ} \mathrm{C}\left(f_{\mathrm{s}} \approx 0.7\right)$ 时, $a_{\dot{\varepsilon}, \mathrm{vp}}$ 和 $m$ 任意假定为 $1.8 \mathrm{MPa}$ 和 0.266 ; 在液相点 $1522{ }^{\circ} \mathrm{C}$ 时 $a_{\dot{\varepsilon}, \mathrm{vp}}$ 和 $m$ 分别 为液体动力学黏度 $5.6 \times 10^{-4} \mathrm{MPa}$ 和 1 .

\section{4 实验及数值模拟结果}

\section{1 微合金低碳钢高温相转变}

图 4 为采用 ThermoCalc 计算的微合金低碳钢 在高温下的各相分数与温度的关系. 在钢凝固过程中, 液相首先析出高温铁素体相 $\delta$; 完全凝固后, 温度低于 $1482{ }^{\circ} \mathrm{C}$ 时, $\delta$ 相开始转变为奥氏体相 $\gamma, 1450{ }^{\circ} \mathrm{C}$ 时 $\delta \rightarrow \gamma$ 相变结束.

\section{2 温度分布}

图 5a 示出了试样沿长度方向的表面温度分布, 离散 点为实验测量值, 曲线为计算值. 该图表明试样长度方向 上存在较大温度梯度. 由于采用了 PID 算法来模拟如图 2 所示升温曲线, 在图 $5 \mathrm{a}$ 中, 可以看到在 $\mathrm{TC} 0$ 位置温度 计算值与测量值完全吻合. 图 $5 \mathrm{~b}$ 为试样中心横截面沿半 径方向的温差 $\left(\theta-\theta_{\mathrm{S}}\right)$ 分布, 离散点为测量值, 曲线为计 算值. 在本实验条件下, 试样径向存在一定温度梯度, 且 随温度升高, 径向温度梯度增大. 


\section{3 高温拉伸实验}

4.3.1 单相奥氏体区拉伸 在本实验条件下, 试样 沿径向及轴向均存在一定的温度梯度. 钢的力学性能参数 通常随温度变化而改变, 因此不均匀温度分布将导致试样 变形及应力分布的不均匀性. 图 6 为 $1300{ }^{\circ} \mathrm{C}$ 时试样内的

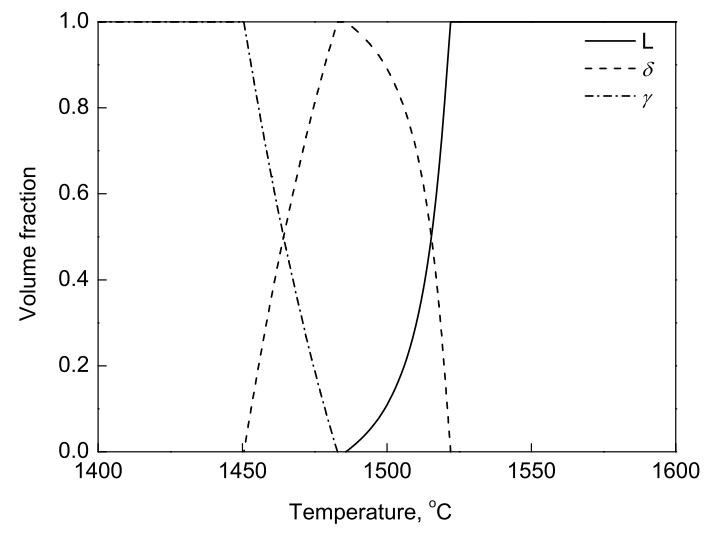

图 4 微合金低碳钢的高温各相分数和温度的关系

Fig.4 Relation between volume fractions of different phases and temperature for micro-alloyed low carbon steel
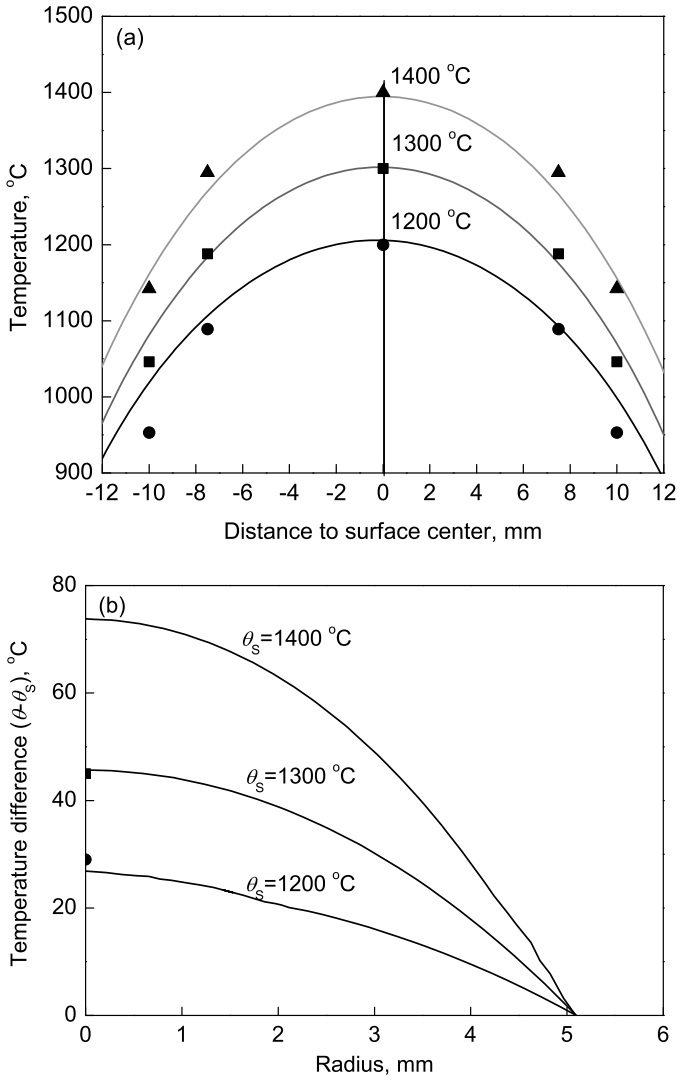

图 5 拉伸试样沿长度方向的表面温度分布及沿半径方向的中 心横截面上的温差 $\left(\theta-\theta_{\mathrm{S}}\right)$ 分布

Fig.5 Surface temperature distribution in the axial direction (a) and radial temperature distribution with respect to surface temperature in the mid-length transverse section (b) in tensile specimen (discrete points are measurements and curves are calculations)
温度分布. 在试样中心横截面上, 轴线中心温度比表面温 度高约 $42{ }^{\circ} \mathrm{C}$, 根据图 4 , 此时试样中心横截面为单一奥氏 体相. 变形速率为 $V_{\mathrm{imp}}=0.01 \mathrm{~mm} / \mathrm{s}$ 时, 在初始拉伸时刻, 试样变形主要集中于中部 $20 \mathrm{~mm}$ 长度的区域内 (图 7), 且变形速率分布极不均匀; 应变速率在轴线中心处达到最 大值, 约 $0.92 \times 10^{-3} \mathrm{~s}^{-1}$, 如图 7 所示. 假设试样均匀变 形区长度为 $l_{0}=10 \mathrm{~mm}$, 则名义应变速率为 $V_{\mathrm{imp}} / l_{0}=1 \times$ $10^{-3} \mathrm{~s}^{-1}$, 高于计算得到的应变速率. 变形不均匀的特 性使名义应变和名义应变速率的计算变得不可靠, 且极不 精确.

图 8 为拉伸 $50 \mathrm{~s}$ 时在中心横截面上的等效应力沿径 向的分布. 可见, 虽然中心横截面内均为奥氏体相, 但应力 沿径向分布仍不均匀, 这是由于奥氏体的力学性质具有温 度依赖性 (式 (31)). 然而应力分布虽然不均, 但相差较小, 约 $0.5 \mathrm{MPa}$ (约 $0.5 / 8 \approx 6.3 \%$ ). 将轴向应力 $\left(\sigma_{z z}\right)$ 沿半 径进行积分可得到拉伸载荷 $F_{\mathrm{cal}}$ (单拉伸状态, $\sigma_{z z}=\bar{\sigma}$ ), 平均应力可由下式计算得到:

$$
\sigma_{z z \text { mean }}=F_{\text {cal }} / S_{0}=\frac{1}{S_{0}} \int_{0}^{r_{0}} \sigma_{z z} 2 \pi r \mathrm{~d} r
$$

计算得到的平均应力 $\sigma_{z z \text {, mean }}=8.28 \mathrm{MPa}$, 该值对 应于图 8 中半径为 $3.5 \mathrm{~mm}$ (即 $r=0.5 \sqrt{2} r_{0}$ ) 处的应力

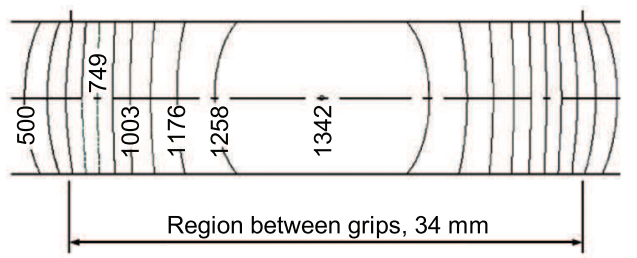

图 6 拉伸试样内的温度分布 (表面中心 $(\mathrm{TC} 0)$ 温度为 $1300{ }^{\circ} \mathrm{C}$ )

Fig.6 Temperature distribution in the central part of tensile specimen at temperature (TC0) of $1300{ }^{\circ} \mathrm{C}$ (unit: ${ }^{\circ} \mathrm{C}$ )

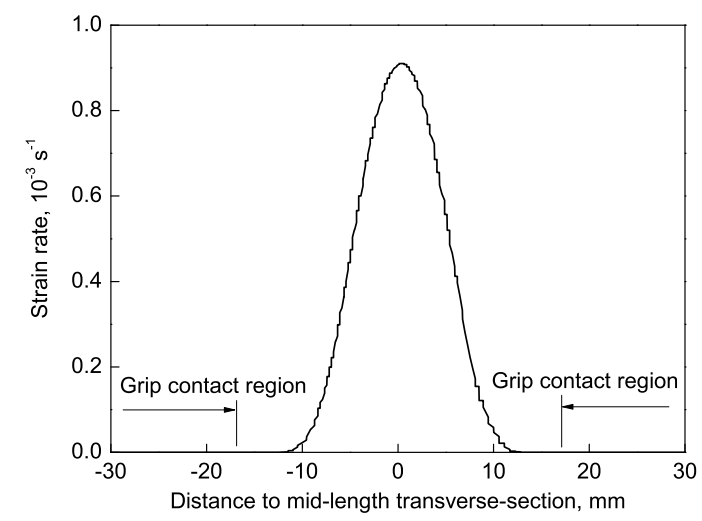

图 7 沿试样轴线方向上的应变速率分布 (时刻 $t_{\mathrm{ms}}$, 变形速率 0.01 $\mathrm{mm} / \mathrm{s}$, 表面中心 $(\mathrm{TC} 0)$ 温度为 $1300{ }^{\circ} \mathrm{C}$ )

Fig.7 Strain rate distribution along axis at the instant $t_{\mathrm{ms}}$, grip velocity of $0.01 \mathrm{~mm} / \mathrm{s}$ and temperature (TC0) of $1300{ }^{\circ} \mathrm{C}$ 
值. 根据以上分析, 当试样中心横截面为单相区时, 应力 分布相对均匀. 当采用基于名义应力及名义应变的简单方 法来研究钢高温力学行为时, 名义应力由实验测量的拉伸 载荷计算 $\sigma_{z z, \mathrm{nom}}=F_{\exp } / S_{0}$, 名义温度则推荐采取中心 横截面 $0.5 \sqrt{2} r_{0}$ 处的温度值, 名义应变及应变速率则需根 据试样实际变形情况假设均匀变形区长度 $l_{0}$.

\subsection{2 多相区域拉伸随着温度升高, 奥氏体相} 开始向高温铁素体相转变 (图 4). 在高温时, 试样内可能 同时存在不同相区域: 单相奥氏体区 $\gamma 、 \gamma+\delta$ 混合区、 单相铁素体区 $\delta$ 及糊状区. 在研究钢在高温糊状区力学 性能时, 一些学者 $[2,23]$ 采用了简单体积平均模型来描述 $(\delta+\gamma)$ 两相混合区力学性能, 即 $w_{i}=g_{i}$. 已有研究 ${ }^{[8,24]}$ 表明在高温条件下高温铁素体相强度大大低于奥氏体相， 因此 Koric 和 Thomas ${ }^{[25]}$ 认为 $(\delta+\gamma)$ 混合区力学性能 不能采用简单体积平均模型来描述, 而是采用: 当 $\delta$ 相分 数 $g_{\delta}$ 小于 0.1 时, 两相区力学性能取决于纯奥氏体相; 而 当 $g_{\delta}$ 大于 0.1 时, 两相区力学性能由纯高温铁素体相确 定. 文献中鲜有报导对奥氏体和高温铁素体双相混合区的 混合法则的研究. 为探讨权函数对拉伸实验的影响, 本文 做了如下 3 种 $w_{i}$ 假设:

$$
\begin{gathered}
w_{\delta}=\left\{\begin{array}{ll}
10 g_{\delta} & \left(g_{\delta}<0.1\right) \\
1 & \left(g_{\delta} \geq 0.1\right)
\end{array}, \quad w_{\gamma}=1-w_{\delta}\right. \\
w_{\delta}=\left\{\begin{array}{ll}
2 g_{\delta} & \left(g_{\delta}<0.5\right) \\
1 & \left(g_{\delta} \geq 0.5\right)
\end{array}, \quad w_{\gamma}=1-w_{\delta}\right. \\
w_{\delta}=g_{\delta}, \quad w_{\gamma}=1-g_{\delta}
\end{gathered}
$$

上述 3 种方法描述了 $\delta$ - 铁素体的相分数对 $(\delta+\gamma)$ 混合区力学性能的影响. 式 (34) 表示当析出的 $\delta$ - 铁素体 相分数达到 $10 \%$ 时, 双相区力学性能由纯铁素体相的力

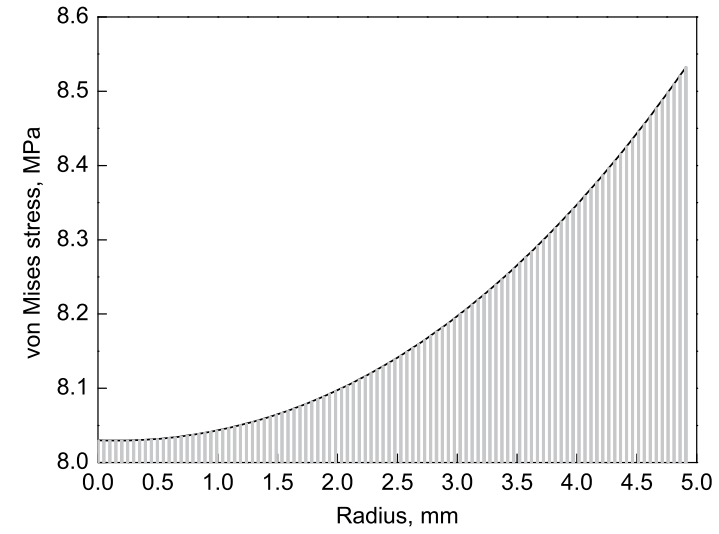

图 8 在中心横截面上等效载荷沿径向分布 (时刻 $t_{\mathrm{ms}}+50 \mathrm{~s}$, 变形 速率 $0.01 \mathrm{~mm} / \mathrm{s}$, 表面中心 $(\mathrm{TC} 0)$ 温度为 $\left.1300{ }^{\circ} \mathrm{C}\right)$

Fig.8 Equivalent stress distribution along radius in midlength transverse section at $50 \mathrm{~s}$ after $t_{\mathrm{ms}}$, grip velocity of $0.01 \mathrm{~mm} / \mathrm{s}$ and temperature (TC0) of $1300{ }^{\circ} \mathrm{C}$
学性能来描述, 即强化 $\delta$ - 铁素体相的软化效应; 式 (36) 为体积平均混合应力模型, 即假定奥氏体相和高温铁素体 相对混合应力具有同等作用; 式 (35) 介于两者之间. 钢 加热时, 以上 3 种方法描述了 $\delta$ - 铁素体相析出对奥氏 体基体相的软化作用; 而在钢凝固冷却时, $\delta$ - 铁素体基体 相内析出奥氏体相, 以上 3 种方法表示了 $\gamma$ 相对 $\delta$ 基 体相的增强作用. 实际相变混合区应力权函数可概括写为 $w_{i}=P^{n}\left(g_{i}\right)$, 即描述为 $g_{i}$ 的 $n$ 次多项式形式, 并应由实 验等方法来确定.

随温度升高, $\delta$ - 铁素体相在奥氏体基体相内析出, 进而试样中心开始熔化. 如图 9 所示, 试样芯部为糊 状区, 最外部为奥氏体区域, 中间为 $(\delta+\gamma)$ 混合两相 区. $1430{ }^{\circ} \mathrm{C}$ 时, 糊状区液相分数在轴线中心处达到最 大值 0.29. 图 10 为在试样中心横截面上沿半径方向 的等效应力分布. 采用式 (34), 等效应力随高温铁素 体相分数增加而迅速降低, 而 $r=4.1-2.8 \mathrm{~mm}$ 两相区 内的应力则变化平缓. 采用体积平均法 (式 (36)) 时, 两相区应力从 $\gamma /(\delta+\gamma)$ 界面至 $\delta /(\delta+\gamma)$ 界面 $(r=$ $2.8 \mathrm{~mm})$ 逐渐降低. 式 (35) 计算的等效应力则介于二者 之间. 图 10 表明试样内复杂的相分布将导致复杂的应 力分布, 这给钢的力学性能研究带来困难, 通过以下的拉 伸载荷分析可以看到这一点. 权函数采用式 (34) 时, 奥 氏体区域 (半径 $4.2-5 \mathrm{~mm}$ ) 承受的拉伸载荷占总载荷 $X_{\gamma}=F_{\gamma} / F_{\text {cal }}=76 \%$, 糊状区载荷分数 $X_{\text {mush }}=8 \%$; 权函 数采用式 $(36)$ 时, $X_{\gamma}$ 约为 $53 \%, X_{\text {mush }}$ 约为 $6 \%$. 在这 2 种情况下, 糊状区对总载荷贡献均很小, 而奥氏体及两 相混合区承载大部分拉伸载荷. 当采用名义应力 - 应变方 法时, 计算得到的名义应力 $\sigma_{z z, \mathrm{nom}}=F_{\exp } / S_{0}$ 小于实际 固相区域应力 $\sigma_{z z \text {,solid }}=F_{\exp }\left(1-X_{\text {mush }}\right) /\left(S_{\operatorname{mix}}+S_{\gamma}\right)$, 又高于糊状区应力 $\sigma_{z z, \text { mush }}=F_{\exp } X_{\text {mush }} / S_{\text {mush }}$. 这表 明当试样内存在复杂相分布情况下, 基于名义应力及应变 的数据处理方法会引起较大的误差, 而采用数值模拟的方 法则不存在这种缺点. 由图 10 可以看出, 相 (分数) 分布 影响金属在近熔点区域内的应力分布, 从而影响铸件热裂 纹缺陷的预测准确性, 这表明了在力学模型中考虑多相力 学模型的必要性.

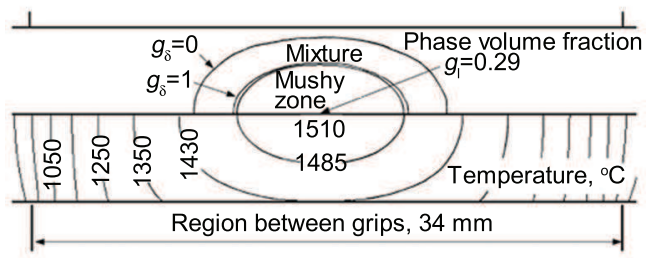

图 9 拉伸试样内的温度与相分数分布 (表面中心温度 (TC0) 为 $1430{ }^{\circ} \mathrm{C}$ )

Fig.9 Distribution of temperature and the volume fraction of $\delta$-ferrite and liquid in the central part of specimen at temperature (TC0) of $1430{ }^{\circ} \mathrm{C}$ (unit: ${ }^{\circ} \mathrm{C}$ ) 


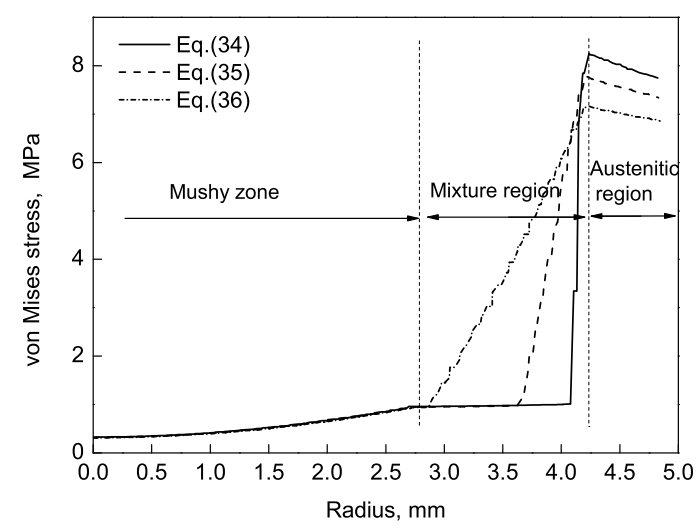

图 10 采用不同应力权函数计算得到的在中心横截面上沿半径 方向的等效应力分布 (表面中心温度 (TC0) 为 $1430{ }^{\circ} \mathrm{C}$, 变形速度 $0.01 \mathrm{~mm} / \mathrm{s}$ )

Fig.10 Radial distribution of equivalent stresses in the midlength transverse section, which are calculated by three weighting methods at temperature (TC0) of $1430{ }^{\circ} \mathrm{C}$ and grip velocity of $0.01 \mathrm{~mm} / \mathrm{s}$

4.3.3 关于本构模型参数的标定 根据上文的分 析, 当由实验测量的载荷 - 位移曲线来标定本构模型的材 料参数时, 采用名义应力 - 应变的简单处理方法会引起较 大误差, 只能采用基于数值模拟的方法. 通过高温力学拉 伸实验, 测量得到的载荷 - 位移曲线用来描述材料的力学 行为. 拉伸实验过程的数值模拟可得到载荷 - 位移曲线, 并与实验测量曲线比较, 通过数值优化算法对本构模型中 材料参数进行标定. 载荷和位移由下式计算:

$$
\begin{gathered}
F(t)=\sum_{\text {contact node }} \vec{T}_{\text {node }}(t) \cdot \vec{x}_{z} 2 \pi r_{\text {node }} l_{\text {node }} \\
\Delta l(t)=\int_{t_{\mathrm{ms}}}^{t} V_{\mathrm{imp}}(t) \mathrm{d} t
\end{gathered}
$$

式中 $\vec{T}_{\text {node }}(t)$ 表示接触节点 node 的表面应力, $r_{\text {node }}$ 为 节点半径, $l_{\text {node }}$ 为节点所在边界长度, $F(t)$ 为拉伸载荷, $\Delta l(t)$ 为试样伸长位移. 图 11 为计算得到的在不同温度 时的载荷 - 位移曲线 (变形速度 $0.01 \mathrm{~mm} / \mathrm{s}$ ), 相应的实 验数据也以离散点的形式标出. 由图可见, 计算的和实验 测量的曲线存在偏差, 实际测量的奥氏体相强度大于计算 值 $\left(1200\right.$ 和 $\left.1300{ }^{\circ} \mathrm{C}\right)$; 当存在复杂多相区时 $\left(1430{ }^{\circ} \mathrm{C}\right.$, 如 $\gamma, \delta, \gamma+\delta$ 及糊状区), 测量载荷小于计算值. 当采用不同 权函数时, 计算的拉伸载荷也不相同, 式 (36) 计算的拉伸 载荷最大, 式 (34) 计算的最小. 该图显示的载荷权函数敏 感性的意义在于, 多相力学模型中的权函数可以通过拉伸 实验及数值反算方法来确定.

研究该低碳钢在高温下的力学行为将涉及到奥氏体 相力学行为, 高温铁素体相力学行为, 两相混合法则以及 糊状区力学行为. 然而前面分析表明, 在高温时, 由于试 样内温度梯度增大, 试样内部存在复杂的相转变区以及糊

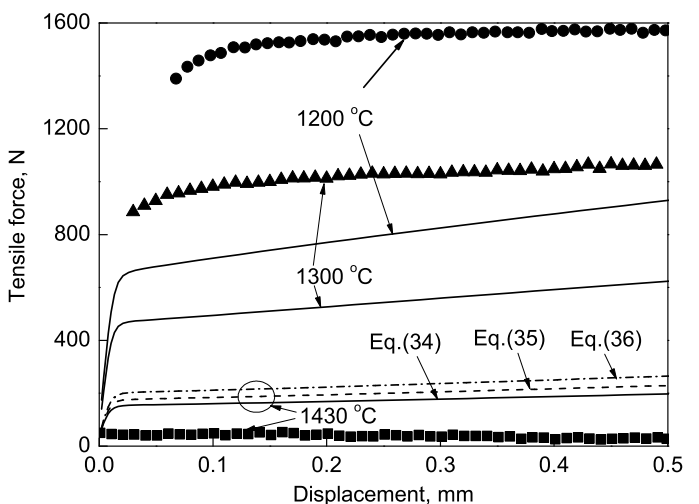

图 11 计算和测量的拉伸载荷的比较 (变形速度 $0.01 \mathrm{~mm} / \mathrm{s}$, 表 面中心温度 $(\mathrm{TC} 0) 1430{ }^{\circ} \mathrm{C}$, 离散点为测量数据, 曲线为 计算值)

Fig.11 Comparison between the experimentally measured and the calculated tensile forces with imposed grip velocity of $0.01 \mathrm{~mm} / \mathrm{s}$ at temperature (TC0) of $1430{ }^{\circ} \mathrm{C}$ (discrete points: experiments; curves: calculations)

状区, 使应力应变分布不均匀, 这使得基于名义应力 - 应 变的本构方程材料参数的标定方法变得不可行. 因此在存 在温度梯度的条件下, 研究钢的高温力学本构模型需要采 用数值方法. 数值模拟算法要求能够得到稳定可靠的计算 结果; 数值优化算法, 其本质是通过不断减少计算和测量 的载荷 - 位移曲线之间的偏差, 最终得到优化的本构方程 的材料参数值. 本文所建立的电势 - 热 - 力耦合计算模 型考虑了固态相变及糊状区的力学行为, 为微合金低碳钢 高温本构方程材料参数的标定构建了稳定可靠的数值模 拟算法.

\section{5 结论}

（1）建立了针对复杂热 - 载荷历史的高温拉伸实验 的电势 - 热 - 力耦合有限元计算模型. 该计算模型采用 PID 算法来实现试样按预设曲线进行加热的过程, 可以 对变形速率随时间变化的拉伸实验进行数值模拟. 建立 的力学求解模型考虑了微合金低碳钢在高温条件下发生 的奥氏体相和高温铁素体相的 $\gamma / \delta$ 相变, 建立了多相区 混合力学模型, 描述了 $(\gamma+\delta)$ 两相混合区的力学行为, 从而使该力学求解模型更加精确地描述了微合金低碳钢 在高温条件下的力学行为.

(2) 实验及数值模拟结果表明, 本实验条件下试样中 存在较大的轴向及径向温度梯度. 在 $1300{ }^{\circ} \mathrm{C}$ 时, 试样中 心横截面单相奥氏体区内径向应力分布相差仅 $6.3 \%$; 在 $1430{ }^{\circ} \mathrm{C}$ 时, 试样内中心横截面内同时存在单相奥氏体 $\gamma$ 区、混合两相 $(\gamma+\delta)$ 区、单相高温铁素体 $\delta$ 区和糊状区, 应力分布极不均匀. 在进行微合金低碳钢高温力学本构方 程材料参数的标定时, 基于名义应力应变的简单方法会引 起较大误差, 而基于数值模拟的优化反算方法则是十分有 效及准确的. 


\section{参考文献}

[1] Eskin D G, Katgerman L. Metall Mater Trans, 2007; 38A: 1511

[2] Won Y M, Yeo T J, Seol D J, Oh K H. Metall Mater Trans, 2000; 31B: 779

[3] Bellet M, Cerri O, Bobadilla M, Chastel Y. Metall Mater Trans, 2009; 40A: 2705

[4] Rappaz M, Drezet J M, Gremaud M. Metall Mater Trans, 1999; 30A: 449

[5] Suzuki M, Yu C H, Emi T. ISIJ Int, 1997; 37: 375

[6] Bernhard C, Xia G. Ironmaking Steelmaking, 2006; 33: 52

[7] Kim K, Oh K H, Lee D N. Scr Mater, 1996; 34: 301

[8] Seol D J, Won Y M, Yeo T J, Oh K H, Park J K. ISIJ Int, 1999; 39: 91

[9] Seol D J, Won Y M, Oh K H, Shin Y C, Yim C H. ISIJ Int, 2000; 40: 356

[10] Brown S G R, James J D, Spittle J A. Model Simul Mater Sci Eng, 1997; 5: 539

[11] Norris S D, Wilson I. Model Simul Mater Sci Eng, 1999; 7: 297

[12] Solek K, Mitura Z, Kuziak R. In: K J Bathe ed., Proc 3rd MIT Conf Computational Fluid and Solid Mechanics, Massachusetts Institute of Technology: Cambridge, 2005: 1001

[13] Rappaz M, Bellet M, Deville M. Numerical Modeling in
Materials Science and Engineering. London: Springer, 2003: 28

[14] Bellet M, Fachinotti V D. Comput Meth Appl Mech Eng, 2004; 193: 4355

[15] Thomas B G, Bellet M. ASM Handbook, Volume 15, Casting Division 4: Modeling and Analysis of Casting Processes, OH: ASM International, 2008; 15: 449

[16] Bellet M, Heinrich A. ISIJ Int, 2004; 44: 1686

[17] Bellet M, Decultieux F, Menai M, Bay F, Levaillant C, Chenot J L, Schmidt P, Svensson I L. Metall Mater Trans, 1996; 27B: 81

[18] Lee M G, Kim S J, Han H N, Jeong W C. Int J Plast, 2009; 25: 1726

[19] Ankem S, Margolin H, Greene C A, Neuberger B W, Oberson P G. Prog Mater Sci, 2006; 51: 632

[20] Wray P J. Met Technol, 1981; 8: 466

[21] Harste K, Schwerdtfeger K. Mater Sci Technol, 1996; 12: 378

[22] Kozlowski P F, Thomas B G, Azzi J A, Hao W. Metall Trans, 1992; 23A: 903

[23] Mizukami H, Shirai Y, Yamanaka A. ISIJ Int, 2006; 46: 1040

[24] Wray P J. Metall Trans, 1976; 7A: 1621

[25] Koric S, Thomas B G. J Mater Process Technol, 2008; 197: 408 